\title{
RADAR SIGNAL DETECTION IN THE PRESENCE OF THE ClUTTER WITH PARETO INTENSITY
}

\author{
Abdolreza Mohammadi \\ Department of Electrical Engineering, University of Bojnord, Bojnord, Iran
}

\begin{abstract}
In this paper, we consider the problem of radar signal detection in the presence of the clutter with Pareto distribution intensity. The clutter is modelled using compound Gaussian model with inverse Gamma distribution for its texture. The Swerling 1 model with an unknown Doppler shift is considered for the target signal. We obtain the optimum detector based on the Neyman-Pearson criteria for completely known target. Moreover, a sub-optimum detector using weak signal approximation and averaging oversignal's distribution is proposed for target with Swerling 1 model with an unknown Doppler shift.
\end{abstract}

\section{KEYWORDS}

Compound Gaussian model, Inverse Gamma texture, Swerling 1 model, Unknown Doppler shift.

\section{INTRODUCTION}

A fundamental task of radar systems is signal detection. The problem of detection of the nonfluctuating targets in white Gaussian noise was considered by Marcum in 1947 [1]. In 1960,Swerling [2] presented models for various targets based on their fluctuation. Researchers have considered Gaussian and non-Gaussian models for clutter in the literature [3]. The nonGaussian clutter often is modelled using compound Gaussian model which is used for high resolution radars [4]. In the compound Gaussian model, the clutter is modelled as a correlated complex Gaussian process with its power being a positive random variable referred to as the "texture" [5]. Therefore, the distribution of the texture determines the distribution of the clutter. For example, a texture with Gamma distribution produce a $\mathrm{K}$ distributed clutter while a clutter with Pareto intensity has a texture with inverse Gamma distribution [6].Experimental data in high resolution with low grazing angle radars show that the compound Gaussian model with inverse Gamma texture is more suitable for sea and ocean clutter [7,8].

In recent years, many studies have been devoted on the detection of radar signal and many approaches are provided for detecting the target signals. In [9], the authors have provided a comprehensive survey of radar detection in the compound Gaussian clutter, particularly in the context of coherent approaches. Farina and Lombardo in [10] has been modelled a mixture of Kdistributed and Gaussian clutters using mathematical framework. A generalized likelihood ratio test (GLRT) for the problem of radar signal detection in the presence of the K distributed clutter has been suggested by Conte in [11].

An order statistic decision rule in the clutter with the spatially correlated Pareto distribution has presented by Weinberg in [6]. In [12], a distributed target detector based on the two-step GLRT

DOI: 10.14810/elelij.2018.7202 
for high resolution radar has been proposed and its performance has been evaluated. In [13] several sub-optimum detectors has proposed for a perfectly known target based on the maximum likelihood (ML) and maximum a posteriori (MAP)estimations of a texture parameter. Moreover, a GLRT for a Rayleigh fluctuating amplitude target with a known Doppler shift has considered in [13]. Shang and Song in [14] have been considered the problem of radar signal detection based on compound Gaussian model with inverse Gamma texture. They proposed one-step generalised likelihood ratio test (1S-GLRT), maximum a posteriori GLRT (MAPGLRT) and two-step GLRT (2S-GLRT) in their work.

In this paper, we investigate the problem of radar signal detection in the presence of compound Gaussian clutter with inverse Gamma texture. We model the received signal using the Swerling 1 model with an unknown Doppler shift in which the fluctuation in the radar echoes is scan to scan [2].We adopt the Neyman-Pearson criteria to find the optimum detector. First, for a completely known target, we obtain the optimum detector. Then, for unknown target, we find the suboptimum detector using weak signal approximation and averaging over signal`s distribution.

The rest of the paper is organized as follows. In section 2, we describe the system model and the basic assumptions about the radar signal as well as the clutter. In section 3 , we obtain the detectors. The simulation is presented in section 4 and the conclusion is drawn in section 5 .

\section{SIGNAL AND CLUTTER MODELS}

Signal detection problem in radar system is modelled by a binary hypothesis test as follows:

$$
\left\{\begin{array}{c}
H_{0}: \boldsymbol{y}=\boldsymbol{n} \\
H_{1}: \boldsymbol{y}=\boldsymbol{s}+\boldsymbol{n}
\end{array}\right.
$$

Where $H_{0}$ and $H_{1}$ are the hypotheses of the absence and presence of the target, respectively. srepresentsthe signal returned from the target and $\boldsymbol{n}$ is the clutter. $y=[y(0), y(1), \ldots, y(N-$ 1) $]^{T}$ denotes the $\mathrm{N}$-dimensional received data and $(.)^{T}$ is the transpose operation.In the following, both signal and clutter models are presented.

\subsection{Clutter Model}

In this paper, we consider a non-Gaussian clutter which can be presented by compound Gaussian model as follows:

$$
\boldsymbol{n}=\sqrt{\tau} \boldsymbol{c}
$$

where $\tau$ (texture) is a positive random variable with probability distribution function (pdf) $f_{\tau}(\tau)$ that represents therandom power of the clutter in the resolution cell and $c$ is a zero mean complex Gaussian random vector with covariance matrix $\boldsymbol{M}$.

The joint pdf of the clutter can be written as [14]:

$$
f_{\boldsymbol{n}}(\boldsymbol{n})=\int_{0}^{\infty} \frac{1}{\pi^{N}|M| \tau^{N}} \exp \left(\frac{\boldsymbol{n}^{H} M^{-1} \boldsymbol{n}}{\tau}\right) f_{\tau}(\tau) d \tau
$$

where $(.)^{H}$ is the hermitian transpose operation. In this paper, we consider inverse Gamma pdf for texture as follows [15]:

$$
f_{\tau}(\tau)=\frac{1}{\beta^{\alpha} \Gamma(\alpha)} \tau^{-(\alpha+1)} \exp \left(-\frac{1}{\beta \tau}\right)
$$


where $\Gamma($.$) denotes the Gamma function, \alpha$ and $\beta$ are the shape and scale parameters of the pdf, respectively. Substituting (4) in (3) results in [15]:

$$
f_{\boldsymbol{n}}(\boldsymbol{n})=\frac{\Gamma(\alpha+N)}{\pi^{N}|M| \beta^{\alpha} \Gamma(\alpha)}\left(\frac{1}{\beta}+\boldsymbol{n}^{H} M^{-1} \boldsymbol{n}\right)^{-(\alpha+N)}
$$

\subsection{Signal Model}

In this paper, we assume Swerling 1 model with an unknown Doppler shift for signal as follows:

$$
\boldsymbol{s}=A e^{j \phi} \boldsymbol{\delta}
$$

where $\boldsymbol{\delta}=\left[1, e^{j \Omega}, e^{j 2 \Omega}, \ldots, e^{j(N-1) \Omega}\right]^{T}, \phi$ is the unknown signal phase which is considered uniformly distributed over $[0,2 \pi) . \Omega$ is the Doppler frequency normalized to the radarPulse Repetition Frequency (PRF) which is considered uniformly distributed over $[0,2 \pi)$. Moreover, $A$ is the signal amplitude that is Rayleigh distributed random variable with the power $P_{S}$ as follows:

$$
f_{A}(A)=\frac{2 A}{P_{S}} \exp \left(-\frac{A^{2}}{P_{S}}\right), A \geq 0
$$

\section{DETECTORS}

In this section we obtain proposed detector for our model. First, for completely known signal the optimal detector based on the Neyman-Pearson criteria is determined and then considering Swerlig 1 model for target, a sub-optimal detector is proposed.

\subsection{Optimum Detector}

The optimum detector based on the Neyman-Pearson criteria is equivalent to the comparison of the likelihood ratio test (LRT) to a threshold that is determined based on the desired probability of false alarm i.e.:

$$
\Lambda(\boldsymbol{y} \mid \boldsymbol{s})=\frac{f_{\boldsymbol{y}}\left(\boldsymbol{y} \mid H_{1}\right)}{f_{\boldsymbol{y}}\left(\boldsymbol{y} \mid H_{0}\right)}=\frac{f_{\boldsymbol{n}}(\boldsymbol{y}-\boldsymbol{s})}{f_{\boldsymbol{n}}(\boldsymbol{y})} \underset{H_{1}}{\stackrel{H_{1}}{\gtrless}} T_{o}
$$

Substituting (5) in (8), we have:

$$
\Lambda(\boldsymbol{y} \mid \boldsymbol{s})=\left(\frac{1+\beta \boldsymbol{y}^{H} M^{-1} \boldsymbol{y}}{1+\beta(\boldsymbol{y}-\boldsymbol{s})^{H} M^{-1}(\boldsymbol{y}-\boldsymbol{s})}\right)^{(\alpha+N)} \stackrel{H_{1}}{\underset{H_{0}}{\gtrless} T_{o}}
$$

Therefore, the optimum detector for completely known signal is obtained as follows:

$$
\frac{1+\beta \boldsymbol{y}^{H} M^{-1} \boldsymbol{y}}{1+\beta(\boldsymbol{y}-\boldsymbol{s})^{H} M^{-1}(\boldsymbol{y}-\boldsymbol{s})} \underset{H_{0}}{\stackrel{H_{1}}{\gtrless}} \sqrt[(\alpha+N)]{T_{o}}
$$

\subsection{Sub-Optimum Detector}

The optimum detector for target with Swerling 1 model using (9) is as follows: 
Electrical and Electronics Engineering: An International Journal (ELELIJ) Vol.7, No.1/2, May 2018

$$
\Lambda(\boldsymbol{y})=\int_{\Gamma_{s}} \Lambda(\boldsymbol{y} \mid \boldsymbol{s}) f_{\boldsymbol{s}}(\boldsymbol{s}) d \boldsymbol{s}=\int_{\Gamma_{s}}\left(\frac{1+\beta \boldsymbol{y}^{H} M^{-1} \boldsymbol{y}}{1+\beta(\boldsymbol{y}-\boldsymbol{s})^{H} M^{-1}(\boldsymbol{y}-\boldsymbol{s})}\right)^{(\alpha+N)} f_{\boldsymbol{s}}(\boldsymbol{s}) d \boldsymbol{s}
$$

where $f_{\boldsymbol{s}}(\boldsymbol{s})$ and $\Gamma_{s}$ are the joint pdf and the parameter space of the signal, respectively. Since (11) is an intractable integral, we use some approximation to propose a sub-optimum detector. By defining $\rho_{1}=\boldsymbol{\delta}^{H} M^{-1} \boldsymbol{\delta}, \rho_{2}=\left|\boldsymbol{y}^{H} M^{-1} \boldsymbol{\delta}\right|, \rho_{3}=\boldsymbol{y}^{H} M^{-1} \boldsymbol{y}$, and $\theta=\angle\left(\boldsymbol{y}^{H} M^{-1} \boldsymbol{\delta}\right)$, we have:

$$
(\boldsymbol{y}-\boldsymbol{s})^{H} M^{-1}(\boldsymbol{y}-\boldsymbol{s})=A^{2} \rho_{1}-2 \rho_{2} A \cos (\phi+\theta)+\rho_{3}
$$

Substituting (12) in (9), we have:

$$
\Lambda(\boldsymbol{y} \mid \boldsymbol{s})=\frac{1}{\left(1+\frac{\beta A^{2} \rho_{1}-2 \beta \rho_{2} A \cos (\phi+\theta)}{1+\beta \rho_{3}}\right)^{(\alpha+N)}} \stackrel{H_{1}}{\gtrless} T_{0}
$$

For weak signal with small amplitude $(A \ll 1)$, we can use the following approximation:

$$
\left(1+\frac{\beta A^{2} \rho_{1}-2 \beta \rho_{2} A \cos (\phi+\theta)}{1+\beta \rho_{3}}\right)^{(\alpha+N)} \cong 1+(\alpha+N) \frac{\beta A^{2} \rho_{1}-2 \beta \rho_{2} A \cos (\phi+\theta)}{1+\beta \rho_{3}}
$$

Substituting (14) in (13) and using (6) and weak signal approximation, we have:

$$
\mathcal{W}(\boldsymbol{y} \mid \boldsymbol{s})=\mathcal{W}(\boldsymbol{y} \mid A, \boldsymbol{\phi}, \Omega)=1-(\alpha+N) \frac{\beta A^{2} \rho_{1}-2 \beta \rho_{2} A \cos (\phi+\theta)}{1+\beta \rho_{3}}
$$

Therefore, for random signal, (15) can be written as:

$\mathcal{W}(\boldsymbol{y})=\int_{\Gamma_{s}} \mathcal{W}(\boldsymbol{y} \mid A, \phi, \Omega) f_{\boldsymbol{s}}(\boldsymbol{s}) d \boldsymbol{s}=\int_{\Gamma_{s}}\left(1-(\alpha+N) \frac{\beta A^{2} \rho_{1}-2 \beta \rho_{2} A \cos (\phi+\theta)}{1+\beta \rho_{3}}\right) f_{\boldsymbol{s}}(\boldsymbol{s}) d \boldsymbol{s}(16)$

Integrating over $\phi$, result in:

$$
\mathcal{W}(\boldsymbol{y} \mid A, \Omega)=\frac{1}{2 \pi} \int_{0}^{2 \pi} \mathcal{W}(\boldsymbol{y} \mid A, \phi, \Omega) d \phi=1-(\alpha+N) \frac{\beta A^{2} \rho_{1}}{1+\beta \rho_{3}}
$$

Assuming Rayleigh distribution for $A$, we have:

$$
\mathcal{W}(\boldsymbol{y} \mid \Omega)=\int_{0}^{\infty} \mathcal{W}(\boldsymbol{y} \mid A, \Omega) f_{A}(A) d A=1-P_{S}(\alpha+N) \frac{\beta \rho_{1}}{1+\beta \rho_{3}}
$$

By integrating over $\Omega$ for unknown Doppler shift and using $\frac{1}{2 \pi} \int_{0}^{2 \pi} \boldsymbol{\delta}^{H} M^{-1} \boldsymbol{\delta} d \Omega=\operatorname{trace}\left(M^{-1}\right)$, the sub optimum detector is obtained as follows:

$$
\mathcal{W}(\boldsymbol{y})=\frac{1}{2 \pi} \int_{0}^{2 \pi} \mathcal{W}(\boldsymbol{y} \mid \Omega) d \Omega=1-\frac{\beta \operatorname{trace}\left(M^{-1}\right) P_{S}(\alpha+N)}{1+\beta \rho_{3}} \underset{H_{0}}{\stackrel{H_{1}}{\gtrless} T_{1}}
$$

Therefore, the following detector is obtained:

$$
\begin{gathered}
\boldsymbol{H}_{1} \\
M^{-1} \boldsymbol{y} \underset{H_{0}}{\gtrless} T_{2}
\end{gathered}
$$


where $T_{2}=\frac{\beta \operatorname{trace}\left(M^{-1}\right) P_{S}(\alpha+N)+T_{1}-1}{\beta\left(1-T_{1}\right)}$ is its threshold.

\section{SIMULATION RESULTS}

In this section, numerical examples are provided to evaluate the performance of the proposed detector using the Receiver Operating Characteristic (ROC) curve. Fig. (1) shows the ROC curves for $N=5, S N R=-10, \alpha=2$, and $\beta=1$ for the proposed detector and an energy detector when the target has Swerling 1 model and the optimum detector for completely known signal. As can be seen from this figure, the optimum detector has the best performance for completely known signal. Moreover, the proposed detector outperforms the energy detector for random signal.

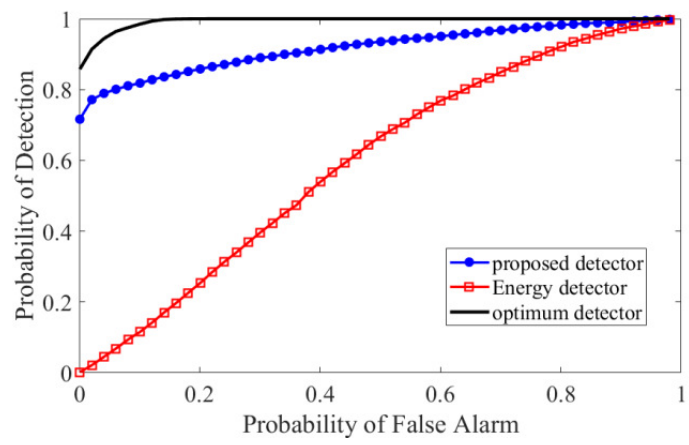

Figure 1.ROC curves for the proposed, optimum and the energy detectors: $N=5, S N R=-10, \alpha=2$, and

$$
\beta=1 \text {. }
$$

\section{CONCLUSiOnS}

In this work, the problem of radar signal detection with unknown Doppler shift in the presence of the clutter with Pareto distribution intensity was considered. Using compound Gaussian model with inverse Gamma texture, the clutter was presented. The optimum detector based on the Neyman-Pearson lemma for completely known signal was obtained. Moreover, a sub optimum detector was proposed for target with Swerling 1 model. Simulation results showed that the proposed detector has a good performance.

\section{ACKNOWLEDGEMENTS}

This research was in part supported by a grant from University of Bojnord( NO. 97/367/730).

\section{REFERENCES}

[1] J. Marcum, "A statistical theory of target detection by pulsed radar," IRE Trans. Inform. Theory, vol. 6, no. 2, pp. 59-267,Apr. 1960.

[2] P. Swerling, "Probability of detection for fluctuating targets," IRE Trans. Inform. Theory, vol. 6, no. 2, pp. 269-308, Apr.1960.

[3] A. D. Maio and M. S. Greco, Modern Radar Detection Theory. SciTech Publishing, 2016.

[4] M. I. Skolnik, Introduction to Radar Systems. McGraw-Hill, 2002.

[5] K. Yao, "A representation theorem and its applications to spherically-invariant random processes," IEEE Trans. Inform. Theory, vol. 19, no. 5, pp. 600-608, Sep. 1973. 
Electrical and Electronics Engineering: An International Journal (ELELIJ) Vol.7, No.1/2, May 2018

[6] G. V. Weinberg, "Non-coherent radar detection in correlated Pareto distributed clutter," IEEE Trans. Aerosp. Electron.Syst., vol. 53, no. 5, pp. 2628-2636, Oct. 2017.

[7] G. V. Weinberg, "Coherent multilook detection for targets in Pareto distributed clutter," Electron. Lett., vol. 47, no. 14, pp. 822-824, Jul. 2011.

[8] G. V. Weinberg, "Assessing detector performance, with application toPareto coherent multilook radar detection,” IET Radar Sonar Navig., vol. 7, no. 4, pp. 401-412, Aug. 2013.

[9] K. J. Sangston and A. Farina, "Coherent radar detection in Compound-Gaussian clutter: Clairvoyant detectors," IEEE Aerosp. Electron. Syst. Mag., vol. 31, no. 11, pp. 42-63, Nov. 2016.

[10] A. Farina and P. Lombardo, "Modelling of a mixture of K-distributed and Gaussian clutter for coherent radar detection,” Electron.Lett., vol. 30, no. 6, pp. 520-521, March 1994.

[11] E. Conte, M. Longo, M. Lops, and S. Ullo, "Radar detection of signals with unknown parameters in K-distributed clutter,” IEE Proc. Radar Signal Process., vol. 138, no. 2, pp. 131-138, Apr. 1991.

[12] Y. Gao, G. Liao, and W. Liu, "High-resolution radar detection in interference and non-homogeneous noise,” IEEE Signal Process. Lett., vol. 23, no. 10, pp. 1359-1363, Oct. 2016.

[13] F. Gini, "Sub-optimum coherent radar detection in a mixture of K-distributed and Gaussian clutter," IEE Proc. Radar,Sonar Navig., vol. 144, no. 1, pp. 131-138, Feb. 1997.

[14] X. Shang, H. Song, "Radar detection based on compound-Gaussian modelwith inverse gamma texture," IET Radar Sonar Navig., vol. 5, no. 3, pp. 315-321, Jan. 2011.

[15] K. J. Sangston,F. Gini, M. S. Greco "Coherent Radar Target Detection in Heavy-Tailed CompoundGaussian Clutter,” IEEE Trans. Aerosp. Electron.Syst., vol. 48, no. 1, pp. 64-77, Jan. 2012. 